Bull. Austral. Math. Soc.

Vol. 59 (1999) [147-152]

\title{
CIRCUMRADIUS-DIAMETER AND WIDTH-INRADIUS RELATIONS FOR LATTICE CONSTRAINED CONVEX SETS
}

\author{
Poh Wah AwYong and Paul R. ScotT
}

Let $K$ be a planar, compact, convex set with circumradius $R$, diameter $d$, width $w$ and inradius $r$, and containing no points of the integer lattice. We generalise inequalities concerning the 'dual' quantities $(2 R-d)$ and $(w-2 r)$ to rectangular lattices. We then use these results to obtain corresponding inequalities for a planar convex set with two interior lattice points. Finally, we conjecture corresponding results for sets containing one interior lattice point.

\section{INTRODUCTION}

Let $\mathcal{K}^{2}$ denote the set of all planar, compact, convex sets. Let $K$ be a set in $\mathcal{K}^{2}$ with circumradius $R(K)=R$, diameter $d(K)=d$, inradius $r(K)=r$, and width $w(K)=w$. Let $K^{o}$ denote the interior of $K$ and let $\Lambda_{R}(\mathbf{u}, \mathbf{v})$ be a rectangular lattice generated by the vectors $\mathbf{u}=(u, 0)$ and $\mathbf{v}=(0, v), u \leqslant v$. In the case where $u=v=1$, we have the integral lattice, denoted by $\Gamma$. Let $G(K, \Lambda)$ denote the number of points of lattice $\Lambda$ in $K$. A number of results concerning the 'dual' quantities $(2 R-d)$ and $(w-2 r)$ have been obtained by Scott $[\mathbf{2}, \mathbf{3}, \mathbf{4}]$ and Awyong [1]. In particular, Awyong [1] proves

THEOREM 1. Let $K$ be a set in $\mathcal{K}^{2}$ having $G\left(K^{o}, \Gamma\right)=0$. Then

$$
\begin{aligned}
& 2 R-d \leqslant \frac{1}{3}, \\
& w-2 r \leqslant \frac{1}{6}(2+\sqrt{3}),
\end{aligned}
$$

with equality when and only when $K \cong E_{0}$ (Figure 1).

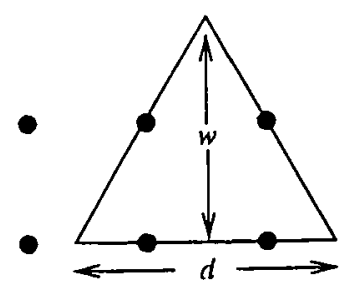

Figure 1: The equilateral triangle $E_{0}$.

Received 28th July, 1998

Copyright Clearance Centre, Inc. Serial-fee code: 0004-9729/99 \$A2.00+0.00. 
The purpose of this paper is to generalise Theorem 1 to rectangular lattices and to use the result to obtain the corresponding inequalities for a set $K \in \mathcal{K}^{2}$ having $G\left(K^{o}, \Gamma\right)=2$. We prove the following results:

THEOREM 2. Let $K$ be a set in $\mathcal{K}^{2}$ with $G\left(K^{o}, \Lambda_{R}\right)=0$. Then

$$
\begin{aligned}
& 2 R-d \leqslant \frac{2}{3}(2-\sqrt{3})\left(\frac{\sqrt{3}}{2} u+v\right) \\
& w-2 r \leqslant \frac{1}{3}\left(\frac{\sqrt{3}}{2} u+v\right)
\end{aligned}
$$

with equality when and only when $K \cong E_{R}$ (Figure 2).

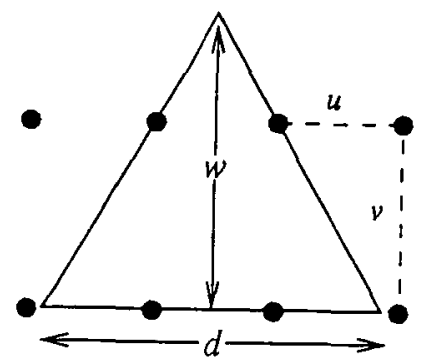

Figure 2: The equilateral triangle $E_{R}$.

Corollary 1 . Let $K$ be a set in $\mathcal{K}^{2}$ with $G\left(K^{o}, \Gamma\right)=2$. Then

$$
\begin{aligned}
& 2 R-d \leqslant \frac{1}{3}(5-2 \sqrt{3}) \approx 0.512, \\
& w-2 r \leqslant \frac{1}{3}\left(2+\frac{\sqrt{3}}{2}\right) \approx 0.955,
\end{aligned}
$$

with equality when and only when $K \cong E_{2}$ (Figure 3 ).

\section{PROOF OF THEOREM 2}

In [1], it was proved that for a set $K \in \mathcal{K}^{2}$,

$$
\begin{aligned}
2 R-d & \leqslant \frac{2}{3}(2-\sqrt{3}) w \\
w-2 r & \leqslant \frac{w}{3}
\end{aligned}
$$




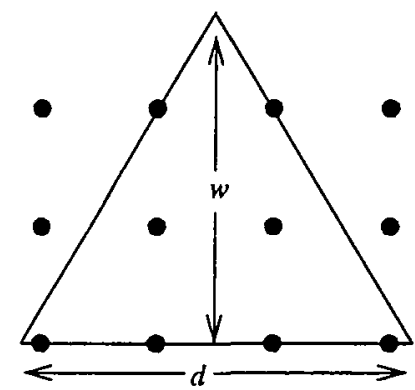

Figure 3: The equilateral triangle $E_{2}$.

with equality when and only when $K$ is an equilateral triangle.

By applying a result by Vassallo [6] to rectangular lattices, we have the result that if $K$ is a set in $\mathcal{K}^{2}$ with $G\left(K^{\circ}, \Lambda_{R}\right)=0$, then

$$
w \leqslant \frac{\sqrt{3}}{2} u+v
$$

Theorem 2 follows immediately by combining inequality (5) with (3) and (4).

\section{Proof of Corollary 1}

Let $K$ now be a set satisfying the conditions of Corollary 1 . Without loss of generality, we may assume that the origin $O$ is one of the lattice points. Let $L$ denote the other lattice point contained in $K^{o}$ and let the coordinates of $L$ be $\left(z_{1}, z_{2}\right)$, where without loss of generality, $z_{1} \geqslant 0, z_{2} \geqslant 0$. By a reflection about $y=x$ if necessary, it suffices to consider those cases for which $z_{1} \geqslant z_{2}$. Since $K^{o}$ contains no other lattice points, the open line segment $O L$ contains no lattice points. Hence we may assume that either $z_{1}=1$ and $z_{2}=0$ or else $z_{1}$ and $z_{2}$ are relatively prime.

If $z_{1}$ and $z_{2}$ are both odd, we consider the sublattice

$$
\Gamma^{\prime}=\{(x, y): x+y \equiv 1(\bmod 2)\}
$$

Clearly, $O \notin \Gamma^{\prime}, L \notin \Gamma^{\prime}$ and $G\left(K^{\circ}, \Gamma^{\prime}\right)=0$. Here we have $u=v=\sqrt{2}$ and by Theorem 2

$$
\begin{aligned}
& 2 R-d \leqslant \frac{1}{3} \sqrt{2} \approx 0.4714<\frac{1}{3}(5-2 \sqrt{3}) \approx 0.512 \\
& w-2 r \leqslant \frac{\sqrt{2}}{3}\left(1+\frac{\sqrt{3}}{2}\right) \approx 0.879<\frac{1}{3}\left(2+\frac{\sqrt{3}}{2}\right) \approx 0.955
\end{aligned}
$$


If $z_{1}$ is odd and $z_{2}$ is even, we consider the sublattice.

$$
\Gamma^{\prime \prime}=\{(x, y): x=m, y=2 n+1, m, n, \in \mathbf{Z}\} .
$$

Clearly $O \notin \Gamma^{\prime \prime}, L \notin \Gamma^{\prime \prime}$ and $G\left(K^{\circ}, \Gamma^{\prime \prime}\right)=0$. In the case where $z_{1}$ is even and $z_{2}$ is odd, we consider the lattice

$$
\Gamma^{\prime \prime \prime}=\{(x, y): x=2 m+1, y=n, m, n \in \mathbf{Z}\} .
$$

Here, we have $G\left(K^{o}, \Gamma^{\prime \prime \prime}\right)=0$. By an appropriate transformation, this is equivalent to the case where $z_{1}$ is odd and $z_{2}$ is even. In this case $u=1$ and $v=2$ and by Theorem 2 , we have

$$
\begin{aligned}
2 R-d \leqslant \frac{1}{3}(5-2 \sqrt{3}) & \approx 0.512, \\
w-2 r \leqslant \frac{1}{3}\left(2+\frac{\sqrt{3}}{2}\right) & \approx 0.955 .
\end{aligned}
$$

Equality is attained when and only when $K \cong E_{2}$ (Figure 3 ).

\section{A Conjecture}

We now conjecture the corresponding inequalities for a set $K$ having $G\left(K^{\circ}, \Gamma\right)=1$. Conjecture. Let $K$ be a set in $\mathcal{K}^{2}$ having $G\left(K^{\circ}, \Gamma\right)=1$. Then

$$
\begin{aligned}
& 2 R-d \leqslant \sqrt{2}\left(\frac{7}{6}-\frac{\sqrt{3}}{2}\right) \approx 0.425, \\
& w-2 r \leqslant \frac{\sqrt{2}}{12}(5+\sqrt{3}) \approx 0.793,
\end{aligned}
$$

with equality when and only when $K \cong E_{1}$ (Figure 4).

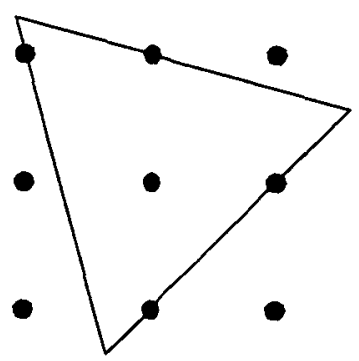

Figure 4: The equilateral triangle $E_{1}$. 
The difficulty which occurs here is that for a set $K$ having $G\left(K^{o}, \Gamma\right)=1, w \leqslant$ $1+\sqrt{2}$, with equality when and only when $K$ is congruent to the isosceles triangle shown in Figure 5 [5]. As this set of largest width is not an equilateral triangle, (3) and (4) do not give sharp inequalities.

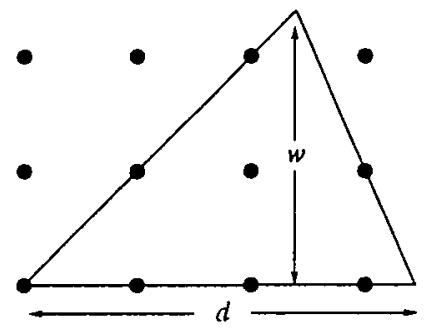

Figure 5: The isosceles triangle $I_{1}$.

A simple calculation shows that the width of $E_{1}$ is $(\sqrt{2}(5+\sqrt{3})) / 4 \approx 2.38$. Hence if $0<w \leqslant(\sqrt{2}(5+\sqrt{3})) / 4$, it follows from (3) and (4) that for this given range of $w$,

$$
\begin{aligned}
& 2 R-d \leqslant \sqrt{2}\left(\frac{7}{6}-\frac{\sqrt{3}}{2}\right) \approx 0.425 \\
& w-2 r \leqslant \frac{\sqrt{2}}{12}(5+\sqrt{3}) \approx 0.793
\end{aligned}
$$

with equality when and only when $K \cong E_{1}$ (Figure 4 ).

This leaves unresolved those cases for which $(\sqrt{2}(5+\sqrt{3})) / 4<w \leqslant 1+\sqrt{2}$. We believe that the set for which $(2 R-d)$ and $(w-2 r)$ are maximal is congruent to the equilateral triangle $E_{1}$ (Figure 4 ).

\section{REFERENCES}

[1] P.W. Awyong, 'An inequality relating the circumradius and diameter of two-dimensional lattice-point-free convex bodies', Amer. Math. Monthly (to appear).

[2] P.R. Scott, 'Two inequalities for convex sets in the plane', Bull. Austral. Math. Soc. 19 (1978), 131-133.

[3] P.R. Scott, 'A family of inequalities for convex sets', Bull. Austral. Math. Soc. 20 (1979), 237-245.

[4] P.R. Scott, 'Sets of constant width and inequalities', Quart. J. Math. Oxford Ser. (2) 32 (1981), 345-348.

[5] P.R. Scott, 'On planar convex sets containing one lattice point', Quart. J. Math. Oxford Ser.(2) 36 (1985), 105-111. 
[6] S. Vassallo, 'A covering problem for plane lattices', Geom. Dedicata 43 (1992), 321-335.

Division of Mathematics

National Institute of Education

469 Bukit Timah Road

Singapore 259756

e-mall: awyongpw@nievax.nie.ac.sg
Department of Pure Mathematics The University of Adelaide

South Australia 5005

e-mail: pscott@maths.adelaide.edu.au 